\title{
Associations of ABCB1, NFKB1, CYP3A, and NR1/2 polymorphisms with cyclosporine trough concentrations in Chinese renal transplant recipients
}

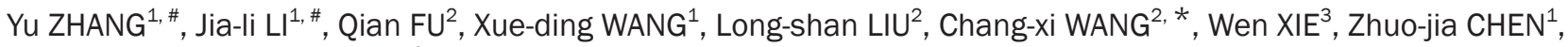 \\ Wen-ying $\mathrm{SHU}^{1}$, Min HUANG ${ }^{1, *}$ \\ ${ }^{1}$ Institute of Clinical Pharmacology, School of Pharmaceutical Sciences, Sun Yat-sen University, Guangzhou 510006, China; ${ }^{2}$ Kidney \\ Transplant Department, Transplant Center, the First Affiliated Hospital of Sun Yat-sen University, Guangzhou 510080, China; ${ }^{3}$ Center \\ for Pharmacogenetics and Department of Pharmaceutical Sciences, University of Pittsburgh, Pittsburgh, PA 15261, USA
}

Aim: Cyclosporine requires close therapeutic drug monitoring because of its narrow therapeutic index and marked inter-individual pharmacokinetic variation. In this study, we investigated the associations of CYP3A4, CYP3A5, ABCB1, NFKB1, and NR1/2 polymorphisms with cyclosporine concentrations in Chinese renal transplant recipients in the early period after renal transplantation. Methods: A total of 101 renal transplant recipients receiving cyclosporine were genotyped for $C Y P 3 A 4 * 1 G, C Y P 3 A 5 * 3, A B C B 1$ C1236T, G2677T/A, C3435T, NFKB1 -94 ins/del ATTG, and NR1/2 polymorphisms. Cyclosporine whole blood levels were measured by a fluorescence polarization immunoassay. Trough concentrations of cyclosporine were determined for days $7-18$ following transplantation.

Results: The dose-adjusted trough concentration $\left(C_{0}\right)$ of cyclosporine in $A B C B 12677$ TT carriers was significantly higher than that in GG carriers together with GT carriers [90.4 24.5 vs $67.8 \pm 26.8(\mathrm{ng} / \mathrm{mL}) /(\mathrm{mg} / \mathrm{kg}), P=0.001]$. ABCB1 $3435 \mathrm{TT}$ carriers had a significantly higher dose-adjusted $C_{0}$ of cyclosporine than CC carriers together with CT carriers $[92.0 \pm 24.0 \mathrm{vs} 68.4 \pm 26.5(\mathrm{ng} / \mathrm{mL}) /(\mathrm{mg} / \mathrm{kg})$, $P=0.002]$. Carriers of the ABCB1 1236TT-2677TT-3435TT haplotype had a considerably higher CsA $C_{0} / D$ than carriers of other genotypes [97.2 \pm 21.8 vs $68.7 \pm 26.9(\mathrm{ng} / \mathrm{mL}) /(\mathrm{mg} / \mathrm{kg}), P=0.001]$. Among non-carriers of the ABCB1 2677 TT and 3435 TT genotypes, patients with the NFKB1 -94 ATTG ins/ins genotype had a significantly higher dose-adjusted $C_{0}$ than those with the -94 ATTG del/del genotype $[75.9 \pm 32.9$ vs $55.1 \pm 15.1(\mathrm{ng} / \mathrm{mL}) /(\mathrm{mg} / \mathrm{kg}), P=0.026]$.

Conclusion: These results illustrate that the ABCB1 and NFKB1 genotypes are closely correlated with cyclosporine trough concentrations, suggesting that these SNPs are useful for determining the appropriate dose of cyclosporine.

Keywords: cyclosporine; ABCB1; NFKB1; NR1I2; CYP3A; genetic polymorphism

Acta Pharmacologica Sinica (2013) 34: 555-560; doi: 10.1038/aps.2012.200; published online 18 Mar 2013

\section{Introduction}

As a member of the calcineurin inhibitor (CNI) family, cyclosporine (CsA) is a first-line immunosuppressant widely used to prevent allograft rejection after solid organ transplantation. However, the bioavailability of cyclosporine ranges from less than $5 \%$ to $89 \%$ in transplant patients ${ }^{[1]}$. Owing to its narrow therapeutic index and marked inter-individual pharmacokinetic variation, therapeutic drug monitoring (TDM) is necessary to adjust the dosage and reduce toxicity ${ }^{[2]}$. Never-

\footnotetext{
\#These authors contributed equally to this work.

* To whom correspondence should be addressed.

E-mail huangmin@mail.sysu.edu.cn (Min HUANG); wcx6363@163.com (Chang-xi WANG)

Received 2012-11-23 Accepted 2012-12-20
}

theless, TDM is hysteretic for optimizing efficacy and limiting toxicity of cyclosporine. Therefore, identifying factors that affect the pharmacokinetics of cyclosporine has great potential for improving the safety and efficacy profile.

Cyclosporine is a substrate of cytochrome 3A4 and 3A5 (CYP3A4 and CYP3A5) and P-glycoprotein (P-gp/MDR1, encoded by $A B C B 1)$. Most previous studies have focused on the influence of genetic variants in the genes encoding CYP3A4, CYP3A5, and P-gp on CsA pharmacokinetics but have yielded conflicting results ${ }^{[3]}$. In addition to the differences in study populations, particularly in sample size and ethnicities, there might be other, as-yet undiscovered, genetic factors that influence the expression or function of CYP3A4, CYP3A5 or P-gp to be discovered.

The pregnane $X$ receptor (PXR, encoded by NR1I2) is 
reported to be the key nuclear receptor regulating the expression of CYP3A4, CYP3A5, and $A B C B 1^{[4]}$. Factors affecting the expression or function of PXR, such as single nucleotide polymorphisms (SNPs), may influence the expression of downstream target genes. T25385C, G24113A, C6994T, C4356T, and G7635A have been reported to be associated with CYP3A4 phenotype, activity and content, whereas A11156C has been associated with variable P-gp levels ${ }^{[5-7]}$. A24622T and C24446A in exon1 have been found to be associated with PXR levels in previous studies from our laboratory ${ }^{[8]}$, suggesting that these two SNPs may also be correlated with the expression of CYP3A4, CYP3A5, or ABCB1.

NF- $\mathrm{kB}$, a protein complex found in almost all animal cell types, is a transcription factor critical for inflammatory responses. It has long been observed that inflammatory responses and infections decrease drug metabolism capacity in humans ${ }^{[9,10]}$. The possible effects of NF-KB on enzymes and transporters related to drug metabolism have been studied because NF-KB is a key regulator of inflammation. Gu et al revealed that NF-KB competitively binds to the retinoid $X$ receptor (RXR), thus preventing the PXR-RXR complex from binding to consensus DNA sequences in the regulatory regions of downstream genes, including $C Y P 3 A 4$ and $A B C B 1^{[11]}$. The NFKB1 gene encodes the p50 subunit of NF- $\mathrm{KB}$, which complexes with p65 to produce the major form of NF- $\mathrm{kB}$, and possesses a functional common insertion/deletion (-94 ins/del ATTG) mutation in its promoter region. Deletion of the ATTG alleles may lead to the loss of binding to nuclear proteins and reduced promoter activity and hence decreased production of the NF-kB p50 isoform ${ }^{[12]}$.

The aim of this study was to comprehensively evaluate the influence of SNPs in CYP3A4, CYP3A5, ABCB1, NR1I2, and NFKB1 on cyclosporine concentration in a group of Chinese renal transplant recipients during the early stage after transplantation.

\section{Materials and methods Ethics statement}

The study was performed in accordance with the Declaration of Helsinki, and ethical approval for this study was obtained from the ethics committee of the First Affiliated Hospital of Sun Yat-Sen University. Written informed consent was obtained from all subjects.

\section{Patients and therapy}

A total of 101 renal transplant recipients $(70$ men and 31 women) who received a renal transplant between 2005 and 2011 at the Department of Organ Transplant, the First Affiliated Hospital, Sun Yat-sen University were enrolled in this study. The average age was $44.5 \pm 13.0$ years (range $18-75$ years), and the average body weight was $60.4 \pm 10.6 \mathrm{~kg}$.

All patients were maintained on a triple immunosuppressive regimen consisting of cyclosporine (Neoral, Novartis Pharma, Basel, Switzerland) as a calcineurin inhibitor, mycophenolate mofetil (Cellcept; Roche, Basel, Switzerland) as a purine inhibitor and steroids (prednisolone, Guangdong Huanan
Pharmacy Ltd, Dongguan, China). CsA was administered at a starting dose of 4-6 mg/ $\mathrm{kg}$ per day following the surgery and administered twice a day in equal amounts. The daily dosage was then adjusted according to trough concentration $\left(C_{0}\right)$, to achieve a target concentration of $200 \mathrm{ng} / \mathrm{mL}$. The steroid regimen was $1 \mathrm{~g} / \mathrm{d}$ intravenously (IV) administered methylprednisolone at the time of surgery followed by $30 \mathrm{mg} / \mathrm{d}$ of oral prednisolone, which was progressively tapered to $15-20 \mathrm{mg} / \mathrm{d}$ by the end of the first month. Mycophenolate mofetil was given at a dose of $1 \mathrm{~g}$ twice daily (bid) during the first month following surgery. Furthermore, other medications known to affect CsA pharmacokinetics, except for prednisolone, were not administered; these included CYP3A or P-gp inducers and inhibitors, such as calcium channel blockers (diltiazem, nicardipine and verapamil), antiepileptics (phenytoin and carbamazepine), antimycotics (fluconazole and ketoconazole), macrolide antibiotics (erythromycin and clarithromycin) and St John's wort. Patients with abnormal hepatic function and combined organ transplants were also excluded.

\section{Data collection}

Body weight, CsA dosages and whole blood CsA concentrations were recorded on days 7-18 after transplantation, when CsA concentrations were stable or dosages had been unchanged for a period of time. Two-milliliter blood samples for the CsA assay were collected before 8:00 AM, immediately before the morning dose. The samples were assayed using the commercially available CsA whole blood monoclonal antibody fluorescence polarization assay ${ }^{[13]}$. The weight-adjusted CsA dosage ( $\mathrm{mg} / \mathrm{kg}$ per $\mathrm{d}$ ) and the dose-adjusted trough concentration $\left[C_{0} / \mathrm{D},(\mathrm{ng} / \mathrm{mL}) /(\mathrm{mg} / \mathrm{kg})\right.$ per d] were calculated.

\section{DNA extraction and genotyping}

Total genomic DNA was extracted from peripheral leukocytes according to a previously described method ${ }^{[14]}$. CYP3A5*3 (A6986G), CYP3A4*1G (G20230A), ABCB1 C1236T, G2677T/A, and $\mathrm{C} 3435 \mathrm{~T}$ polymorphisms were detected by using previously reported polymerase chain reaction restriction-fragment length polymorphism (PCR-RFLP) methods ${ }^{[15-17]}$. The NFKB1 -94ins/del ATTG polymorphism was detected by TaqMan ${ }^{\circledR}$ SNP Genotyping Assay. Polymorphisms of NR1I2, including T25385C, A24622T, C24446A, G24113A, C4356T, A601G, G7635A, A11156C, and C6994T, were genotyped by direct sequencing $^{[5-8]}$.

\section{Statistical analysis}

The pair-wise linkage disequilibrium (LD) for SNPs was estimated by SHEsis (http:/ / analysis2.bio-x.cn/myAnalysis.php). ABCB1 1236-2677-3435 haplotype analysis was performed by PHASE 2.1. Groups were compared using nonparametric tests. For the analysis of continuous pharmacologic variables, we used patient genotypes as categorical independent variables. The Hardy-Weinberg equilibrium test was performed using an appropriate $\chi^{2}$ test. The Mann-Whitney $U$ test was used for comparisons between two groups, and the KruskalWallis $H$ test was used for comparisons among several groups. 
Statistical analysis was performed using SPSS version 17.0 for Windows (SPSS Inc, Chicago, IL, USA). The results are expressed as the mean \pm standard deviation (SD). A $P$-value less than 0.05 was considered statistically significant.

\section{Results}

\section{Genotypes frequencies}

A total of 101 renal transplant recipients were genotyped for polymorphisms in CYP3A5, CYP3A4, ABCB1, NFKB1, and NR1I2. All mutant allele and genotype frequencies were in agreement with previous reports in Han Chinese populations. No differences were observed between allograft recipients and the healthy population, and no deviations from the HardyWeinberg equilibrium were observed.

CYP3A4*1G was in moderate linkage disequilibrium (LD) with CYP3A5*3 $\left(D^{\prime}=0.63\right)$. A moderately high degree of LD between $A B C B 1 \mathrm{C} 1236 \mathrm{~T}, \mathrm{G} 2677 \mathrm{~T} / \mathrm{A}$, and C3435T was also observed. For C1236T-G2677T/A, G2677T/A-C3435T, and C3435T-C1236T, $D^{\prime}$ was $0.56,0.79$ and 0.72 , respectively, which agreed with previous reports. NR1I2 T25385C and G24113A were in complete linkage disequilibrium $\left(D^{\prime}=1\right)$, whereas NR112 C4356T was strongly linked to A-601G SNP $\left(D^{\prime}=0.785\right)$. No significant linkage was found between other individual combinations of SNPs.

According to the haplotype analysis, the frequencies of the three major haplotypes (1236-2677-3435) of ABCB1 were 32.3\% for TTT, $25.7 \%$ for TGC and $20.4 \%$ for CGC. The remaining haplotypes constituted $21.6 \%$ of the patients' haplotypes.

\section{Association between ABCB1 genotype and CsA dose-adjusted trough blood concentrations}

A significantly higher CsA $C_{0} / \mathrm{D}$ was observed in $A B C B 12677$ TT carriers than in the other 2677 genotype carriers [90.4 \pm 24.5 vs $67.8 \pm 26.8(\mathrm{ng} / \mathrm{mL}) /(\mathrm{mg} / \mathrm{kg}), P=0.001]$ (Figure $1 \mathrm{~A})$.

Similarly, carriers of the ABCB1 3435 TT genotype also had a significantly higher CsA $C_{0} / D$ than carriers of the $3435 \mathrm{CT}$ together with CC genotypes $[92.0 \pm 24.0$ vs $68.4 \pm 26.5$ (ng/mL)/(mg/kg), $P=0.002]$ (Figure 1B).

Although patients with the ABCB1 1236TT genotype tended to have higher CsA $C_{0} / D$ than those with $1236 \mathrm{CC}$ or $1236 \mathrm{CT}$ genotypes, no significant association was observed between the $\mathrm{CsA} C_{0} / \mathrm{D}$ and $A B C B 1 \mathrm{C} 1236 \mathrm{~T}$ genotype $(P=0.097)$.

When combining the effects of $A B C B 1 \mathrm{C} 1236 \mathrm{~T}, \mathrm{G} 2677 \mathrm{~T} / \mathrm{A}$ and C3435T, carriers of the 1236TT-2677TT-3435TT haplotype had a considerably higher $\mathrm{CsA}_{C_{0}} / \mathrm{D}$ than carriers of other genotypes [97.2 \pm 21.8 vs $68.7 \pm 26.9(\mathrm{ng} / \mathrm{mL}) /(\mathrm{mg} / \mathrm{kg}), P=0.001$ ] (Figure 1C).

\section{Association between NFKB1 genotypes and CsA dose-adjusted trough blood concentrations}

Patients with the NFKB1 -94 ATTG ins/ins genotype typically had a higher CsA $C_{0} / \mathrm{D}$ than those with the NFKB1 -94 ATTG $\mathrm{del} / \mathrm{del}$ genotype $[78.5 \pm 32.8$ vs $61.1 \pm 19.4(\mathrm{ng} / \mathrm{mL}) /(\mathrm{mg} / \mathrm{kg})]$, but the difference was not statistically significant $(P=0.069)$ (Figure 2A).
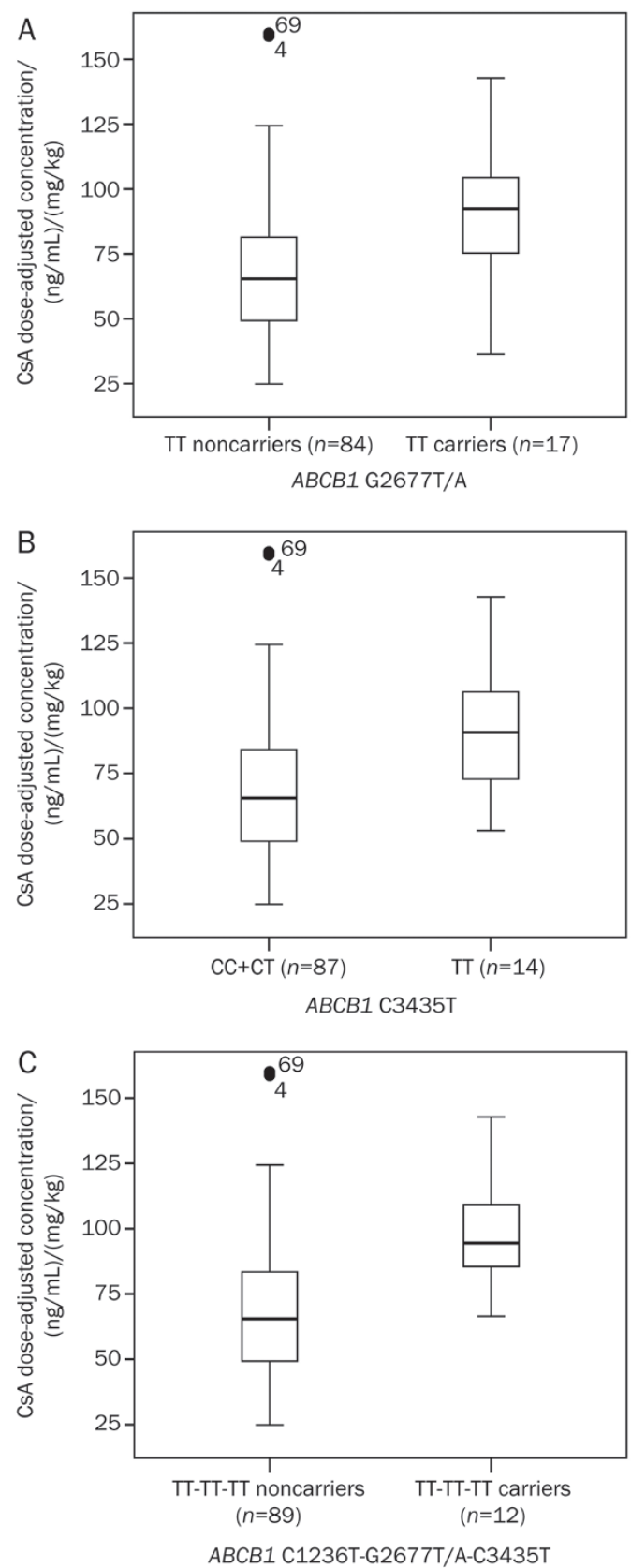

Figure 1. Correlation of the ABCB1 (A) 2677TT genotype, (B) 3435TT genotype and (C) 1236TT-2677TT-3435TT haplotype with CsA $C_{0} / D$ on d 7-18 after transplantation in renal transplant recipients.

Association between CYP3A5, CYP3A4, and NR1/2 genotype and CsA dose-adjusted trough blood concentrations

Carriers of the CYP3A5*1/ ${ }^{*} 1$ and ${ }^{*} 1 /{ }^{*} 3$ genotypes were combined as CYP3A5 expressers, and carriers of the * $3 /{ }^{*} 3$ genotype were defined as non-expressers. No significant difference in $\mathrm{CsA}_{0} / \mathrm{D}$ was observed between CYP3A5 expressers and non-expressers. CYP3A4*1G SNP was also found not to influence $\mathrm{CsA} C_{0} / \mathrm{D}$. When the effects of CYP3A5*3 and CYP3A4* $1 G$ were combined, we were still unable to observe any effect of the CYP3A4-CYP3A5 haplotype on CsA $C_{0} / D$. 

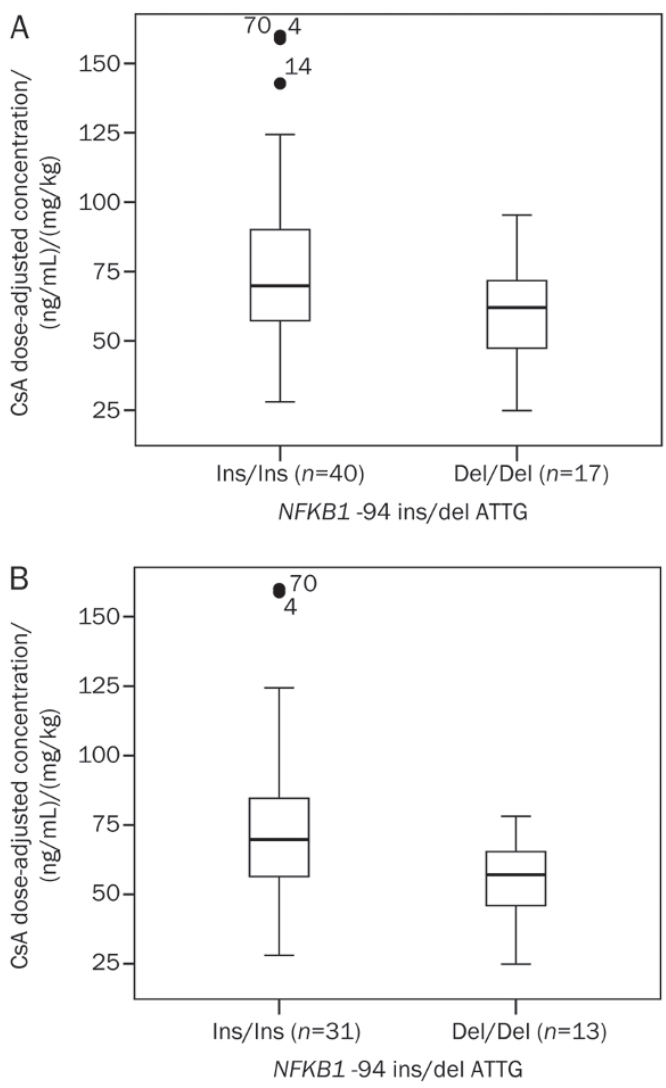

Figure 2. Correlation of NFKB1 genotypes with the dose-adjusted trough concentration of cyclosporine. (A) Influence of the NFKB1 -94 ins/del ATTG genotype on CsA $C_{0} / D$ on d 7-18 after transplantation in renal transplant recipients. (B) Influence of the NFKB1 -94 ins/del ATTG genotype on CsA $C_{0} / D$ on d 7-18 after transplantation in non-carriers of the ABCB1 2677 TT and 3435 TT genotypes.

Although numerous SNPs within the NR1I2 gene were included in this study, no association was observed between CsA $C_{0} /$ D and any of the NR112 SNPs (data not shown).

\section{Stratification analysis}

Stratification analysis was performed to eliminate any confounding effects of the genes that were included in this study. This study was designed to explore an independent effect of CYP3A4, CYP3A5, NFKB1, and NR1I2 genotype on CsA $C_{0} / D$ after 'standardization' for P-gp activity.

Among non-carriers of the $A B C B 12677$ TT genotype and 3435 TT genotype, carriers of the NFKB1 -94 ATTG ins/ins genotype had a significantly higher $\mathrm{CsA} \mathrm{C}_{0} / \mathrm{D}$ than carriers of the -94 ATTG del/del genotype $[75.9 \pm 32.9$ vs $55.1 \pm 15.1$ $(\mathrm{ng} / \mathrm{mL}) /(\mathrm{mg} / \mathrm{kg}), P=0.026]$ (Figure 2B).

\section{Discussion}

The current study is a comprehensive study on polymorphisms of drug metabolic enzymes (CYP3A4 and CYP3A5), a transporter (P-gp) and upstream regulators of them (PXR and NF-kB), to explore the cause of the large inter-individual varia- tion in cyclosporine concentration observed in renal transplant recipients. Moreover, the current study is also directly evaluate the potential effect of NF-KB-related polymorphisms on drug metabolism.

$A B C B 1$ C1236T, G2677T/A, and C3435T were the most commonly and extensively studied SNPs of $A B C B 1$, which can form different haplotypes. Carriers of the TT genotype of ABCB1 C3435T or G2677T/A have been reported to have significantly minimized P-glycoprotein activity compared to wild type activity ${ }^{[18,19]}$, suggesting a higher $\mathrm{CsA}_{0} / \mathrm{D}$ in carriers of the $A B C B 13435$ TT and 2677 TT genotypes.

Separate studies on 44 Caucasian liver transplant recipients ${ }^{[20]}$ and 88 Middle Eastern renal transplant recipients ${ }^{[2]]}$ up to 1 month after transplantation both found carriers of the $A B C B 1$ 3435 TT genotype had a higher $\mathrm{CsA} \mathrm{C}_{0} / \mathrm{D}$ than carriers of 3435 CC or CT genotype. By contrast, in 103 Chinese renal transplant recipients, Qiu et al reported a lower $C_{0} / \mathrm{D}$ in carriers of the $A B C B 1$ 2677GG wild-type genotype than in carriers of the $A B C B 1$ 2677TT variant genotype in the first 8 to $30 \mathrm{~d}$ after transplantation ${ }^{[22,23]}$. Consistent with these studies, we found that the ABCB1 2677TT and 3435TT genotypes and 1236TT2677TT-3435TT haplotype were closely correlated with a higher cyclosporine dose-adjusted $C_{0}$. Moreover, a more obvious influence was found in the 1236TT-2677TT-3435TT haplotype than in each SNP independently; the 1236TT-2677TT-3435TT haplotype contributes more to the variation in CsA concentrations, as determined by multiple linear regression analysis. These findings, which are in agreement with previous observations, suggest that the use of the $A B C B 1$ haplotype is superior to SNP analysis for predicting concentrations of cyclosporine $e^{[24]}$.

As a nuclear factor that takes part in the control of as many as 150 target genes, including many inflammatory genes, NF-KB is a focal point and its related pathways and relationships with many autoimmune diseases and cancers have been widely studied. The majority of studies on NF-KB-related polymorphisms, such as the NFKB1 -94 ins/del ATTG mutation, have been focused on their association with the incidence of tumors and inflammatory diseases ${ }^{[25]}$. Although it has long been observed that inflammatory responses and infections decrease drug metabolism capacity in human, a correlation between NF-kB-related polymorphisms and drug metabolism has never been reported. In a previous in vitro study, Gu et al reported that the structural component of NF-kB, p65, can competitively bind to RXR, which is necessary to form PXRRXR heterodimers. The competitive binding of p65 to RXR, therefore, disrupts the interaction of PXR-RXR heterodimers with the consensus DNA sequences in the regulatory regions of target genes, thus significantly suppressing gene expression $^{[11]}$. It is possible that NF-KB may have an effect on CsA concentrations by suppressing PXR-mediated regulation of CYP3A4/ABCB1. Hence, we speculated that the NFKB1 polymorphism might result in altered NF-kB expression, leading to reduced CYP3A4/ABCB1 expression and increased CsA concentrations.

As reported previously, the del ATTG allele may result in 
decreased NFKB1 transcript levels and therefore decreased p50 protein production ${ }^{[12]}$. Thus, the ins/ins ATTG genotype is speculated to be associated with a higher NF-KB protein content, and consequently lower CYP3A4 expression and ultimately higher CsA concentrations than the levels in carriers of the del/del ATTG genotype. This finding is consistent with our results that carriers of the -94 ATTG ins/ins genotype had a significantly higher $\mathrm{CsA}_{0} / \mathrm{D}$ than carriers of the -94 ATTG del/del genotype in non-carriers of the ABCB1 2677 TT and 3435 TT genotypes. These observations suggest that immunoregulation has a notable effect on CsA concentrations. Further study is needed to assess our speculations and reveal the underlying mechanisms.

Although the importance of CYP3A5*3 in tacrolimus concentrations has been fairly obvious, its influence on CsA concentrations is still in dispute. Several researchers ${ }^{[26-28]}$ have observed a significant association between $C Y P 3 A 5^{*} 3$ and CsA trough concentrations. However, no association between $C Y P 3 A 5^{*} 3$ and CsA concentrations was found in this study, which is in consistent with some previous reports ${ }^{[29-31]}$. CYP3A4*1G is a CYP3A4 SNP with the highest occurrence in Chinese populations, but a definitive function has not been reported. A series of studies examined the relationship between the CYP3A4*1G polymorphism and drug metabolism but resulted in inconsistent findings ${ }^{[32-34]}$. In agreement with our observation, CYP3A4* $1 \mathrm{G}$ was not associated with cyclosporine concentrations in 126 renal recipients ${ }^{[27]}$; however, Qiu et al ${ }^{[22]}$ and Hu et $a l^{[35]}$ found that it was associated with a lower CsA concentration. Differences in study populations, sample size, ethnicities and particularly in the duration after transplantation might be possible confounding factors. During the early stage after transplantation, steroids were used at a high dose, which might have induced CYP3A.

Although they were reported to be functional in previous studies, none of the NR1I2 SNPs were found to be correlated with CsA concentrations in our study ${ }^{[5,6,8]}$. However, most of these studies were in vitro, without the consideration of many possible influences caused by other factors in vivo. Another caveat to be taken into account is the induction of PXR by steroids $^{[36]}$ because the recipients in this study, who were at an early stage after transplantation, routinely received a high dose of steroids. Therefore, the impact of NR1I2 SNPs on cyclosporine concentrations at a stable stage after transplantation in patients who received a lower dose of steroids needs to be studied.

In summary, this study reports the potential effect of NF-kBrelated polymorphisms on clinical drug metabolism. We also found that the $A B C B 1$ 1236TT-2677TT-3435TT haplotype was most significantly correlated with CsA concentrations in Chinese renal transplant recipients. Patients who carry the $A B C B 1$ 1236TT-2677TT-3435TT haplotype may be at a greater risk of high CsA concentrations that could lead to hepatotoxicity and nephrotoxicity. NFKB1 polymorphisms were identified as a minor significant factor associated with CsA concentrations in this study, and patients with the NFKB1 ins/ins ATTG genotype tended to have higher CsA concentrations. We also propose that pre-transplant genotyping of the $A B C B 1 \mathrm{C} 1236 \mathrm{~T}$, G2677T/A, C3435T, and NFKB1 ins/ins ATTG genotypes may be used as an index for CsA dosing in clinical practice and that the initial CsA dose for carriers of the ABCB1 1236TT-2677TT3435TT haplotype and NFKB1 -94 ins/ins ATTG genotype be lower to prevent possible toxicities during the early stage of transplantation.

\section{Acknowledgements}

We appreciate the financial support provided by a National Science and Technology Major Project in Science and Technology grant from the Science and Technology Ministry of China (Grant No 2012ZX09506001-004), National Natural Science Foundations of China (№ 81102515, 81072708, and 81173131) and Medical Research Foundation of Guangdong Province (№ B2011067).

\section{Author contribution}

Yu ZHANG, Jia-li LI, Min HUANG, and Chang-xi WANG designed the research. Yu ZHANG, Jia-li LI, Xue-ding WANG, Qian FU, and Long-shan LIU performed the research. Yu ZHANG, Jia-li LI, and Wen-ying SHU contributed new reagents and analytic tools. Yu ZHANG and Zhuo-jia CHEN analyzed the data. Yu ZHANG, Jia-li LI, and Wen XIE wrote the paper.

\section{References}

1 Ptachcinski RJ, Venkataramanan R, Burckart GJ. Clinical pharmacokinetics of cyclosporin. Clin Pharmacokinet 1986; 11: 107-32.

2 Kahan BD, Keown P, Levy GA, Johnston A. Therapeutic drug monitoring of immunosuppressant drugs in clinical practice. Clin Ther 2002; 24: 330-50.

3 Staatz CE, Goodman LK, Tett SE. Effect of CYP3A and ABCB1 single nucleotide polymorphisms on the pharmacokinetics and pharmacodynamics of calcineurin inhibitors: Part I. Clin Pharmacokinet 2010; 49: $207-21$.

4 Wang $\mathrm{H}$, LeCluyse EL. Role of orphan nuclear receptors in the regulation of drug-metabolising enzymes. Clin Pharmacokinet 2003; 42: $1331-57$.

5 Zhang J, Kuehl P, Green ED, Touchman JW, Watkins PB, Daly A, et al. The human pregnane $X$ receptor: genomic structure and identification and functional characterization of natural allelic variants. Pharmacogenetics 2001; 11: 555-72.

6 Lamba J, Lamba V, Strom S, Venkataramanan R, Schuetz E. Novel single nucleotide polymorphisms in the promoter and intron 1 of human pregnane $X$ receptor/NR112 and their association with CYP3A4 expression. Drug Metab Dispos 2008; 36: 169-81.

7 Wang XD, Deng XY, Chen J, Li JL, Chen X, Zhao LZ, et al. Single nucleotide polymorphisms of the pregnane $X$ receptor gene in Han Chinese and a comparison with other ethnic populations. Pharmacology 2008; 81: 350-4.

8 Wang XD, Li JL, Su QB, Deng XY, Lu Y, Chen J, et al. A pharmacogenetic study of pregnane $X$ receptor (NR112) in Han Chinese. Curr Drug Metab 2007; 8: 778-86.

9 Morgan ET. Regulation of cytochromes P450 during inflammation and infection. Drug Metab Rev 1997; 29: 1129-88.

10 Renton KW. Cytochrome $\mathrm{P} 450$ regulation and drug biotransformation during inflammation and infection. Curr Drug Metab 2004; 5: 
235-43.

$11 \mathrm{Gu}$ X. Role of NF-KB in regulation of PXR-mediated gene expression: A mechanism for the suppression of cytochrome P-450 3A4 by proinflammatory agents. J Biol Chem 2006; 281: 17882-9.

12 Karban AS, Okazaki T, Panhuysen Cl, Gallegos T, Potter JJ, BaileyWilson JE, et al. Functional annotation of a novel NFKB1 promoter polymorphism that increases risk for ulcerative colitis. Hum Mol Genet 2004; 13: 35-45.

13 Sabate I, Ginard M, Gonzalez JM, Baro E, Acebes G, Cuadros J, et al. Evaluation of the AXSYM monoclonal cyclosporin assay and comparison with radioimmunoassay. Ther Drug Monit 2000; 22: 474-80.

14 Loparev VN, Cartas MA, Monken CE, Velpandi A, Srinivasan A. An efficient and simple method of DNA extraction from whole blood and cell lines to identify infectious agents. J Virol Methods 1991; 34: 105-12.

15 Wang Y, Wang C, Li J, Wang X, Zhu G, Chen X, et al. Effect of genetic polymorphisms of CYP3A5 and MDR1 on cyclosporine concentration during the early stage after renal transplantation in Chinese patients co-treated with diltiazem. Eur J Clin Pharmacol 2009; 65: 239-47.

16 Gao Y, Zhang LR, Fu Q. CYP3A4*1G polymorphism is associated with lipid-lowering efficacy of atorvastatin but not of simvastatin. Eur J Clin Pharmacol 2008; 64: 877-82.

17 Senol Tuncay S, Okyay P, Bardakci F. Identification of NF-kB1 and NFKBl $\alpha$ polymorphisms using PCR-RFLP assay in a Turkish population. Biochem Genet 2010; 48: 104-12.

18 Kimchi-Sarfaty C, Oh JM, Kim IW, Sauna ZE, Calcagno AM, Ambudkar $\mathrm{SV}$, et al. A "silent" polymorphism in the MDR1 gene changes substrate specificity. Science 2007; 315: 525-8.

19 Wang D, Johnson AD, Papp AC, Kroetz DL, Sadee W. Multidrug resistance polypeptide 1 (MDR1, ABCB1) variant 3435C >T affects mRNA stability. Pharmacogenet Genomics 2005; 15: 693-704.

20 Bonhomme-Faivre L, Devocelle A, Saliba F, Chatled S, Maccario J, Farinotti R, et al. MDR-1 C3435T polymorphism influences cyclosporine a dose requirement in liver-transplant recipients. Transplantation 2004; 78: 21-5.

21 Azarpira N AM, Behzad-Behbahanie A, Geramizadeh B, Behzadi $\mathrm{S}$, Malekhoseinie SA, Raisjalal GH, et al. Association between cyclosporine concentration and genetic polymorphisms of CYP3A5 and MDR1 during the early stage after renal transplantation. Exp Clin Transplant 2006; 4: 416-9.

22 Qiu XY, Jiao Z, Zhang M, Zhong L, Liang HQ, Ma CL, et al. Association of MDR1, CYP3A4*18B, and CYP3A5*3 polymorphisms with cyclosporine pharmacokinetics in Chinese renal transplant recipients. Eur J Clin Pharmacol 2008; 64: 1069-84.

23 Turolo S, Tirelli AS, Ferraresso M, Ghio L, Belingheri M, Groppali E, et al. Frequencies and roles of CYP3A5, CYP3A4, and ABCB1 single nucleotide polymorphisms in Italian teenagers after kidney transplantation. Pharmacol Rep 2010; 62: 1159-69.

24 Chowbay B, Cumaraswamy S, Cheung YB, Zhou Q, Lee EJ. Genetic polymorphisms in MDR1 and CYP3A4 genes in Asians and the influence of MDR1 haplotypes on cyclosporin disposition in heart transplant recipients. Pharmacogenetics 2003; 13: 89-95.

25 Sun XF, Zhang H. NFKB and NFKBI polymorphisms in relation to susceptibility of tumour and other diseases. Histol Histopathol 2007; 22: 1387-98.

26 Chu XM, Hao HP, Wang GJ, Guo LQ, Min PQ. Influence of CYP3A5 genetic polymorphism on cyclosporine A metabolism and elimination in Chinese renal transplant recipients. Acta Pharmacol Sin 2006; 27: 1504-8.

27 Meng XG, Guo CX, Feng GQ, Zhao YC, Zhou BT, Han JL, et al. Association of CYP3A polymorphisms with the pharmacokinetics of cyclosporine $A$ in early post-renal transplant recipients in China. Acta Pharmacol Sin 2012; 33: 1563-70.

28 Song J, Kim MG, Choi B, Han NY, Yun HY, Yoon JH, et al. CYP3A5 polymorphism effect on cyclosporine pharmacokinetics in living donor renal transplant recipients: analysis by population pharmacokinetics. Ann Pharmacother 2012; 46: 1141-51.

29 Bouamar R, Hesselink DA, van Schaik RH, Weimar W, Macphee IA, de Fijter JW, et al. Polymorphisms in CYP3A5, CYP3A4, and ABCB1 are not associated with cyclosporine pharmacokinetics nor with cyclosporine clinical end points after renal transplantation. Ther Drug Monit 2011; 33: 178-84.

30 Chen B, Zhang W, Gu Z, Li J, Zhang Y, Cai W. Population pharmacokinetic study of cyclosporine in Chinese renal transplant recipients. Eur J Clin Pharmacol 2011; 67: 601-12.

31 Santoro A, Felipe CR, Tedesco-Silva H, Medina-Pestana JO, Struchiner CJ, Ojopi EB, et al. Pharmacogenetics of calcineurin inhibitors in Brazilian renal transplant patients. Pharmacogenomics 2011; 12 : 1293-303.

32 Dong ZL, Li H, Chen QX, Hu Y, Wu SJ, Tang LY, et al. Effect of CYP3A4*1G on the fentanyl consumption for intravenous patientcontrolled analgesia after total abdominal hysterectomy in Chinese Han population. J Clin Pharm Ther 2012; 37: 153-6.

33 Miura M, Satoh S, Kagaya H, Saito M, Numakura K, Tsuchiya N, et al. Impact of the CYP3A4*1G polymorphism and its combination with CYP3A5 genotypes on tacrolimus pharmacokinetics in renal transplant patients. Pharmacogenomics 2011; 12: 977-84.

34 Yuan R, Zhang X, Deng Q, Wu Y, Xiang G. Impact of CYP3A4*1G polymorphism on metabolism of fentanyl in Chinese patients undergoing lower abdominal surgery. Clin Chim Acta 2011; 412: 755-60.

35 Hu YF, Tu JH, Tan ZR, Liu ZQ, Zhou G, He J, et al. Association of CYP3A4*18B polymorphisms with the pharmacokinetics of cyclosporine in healthy subjects. Xenobiotica 2007; 37: 315-27.

36 Miura M, Satoh S, Inoue K, Kagaya H, Saito M, Inoue T, et al. Influence of CYP3A5, ABCB1, and NR1I2 polymorphisms on prednisolone pharmacokinetics in renal transplant recipients. Steroids 2008; 73 : 1052-9. 